\title{
Leucodistrofia Metacromática: relato de caso de criança com forma infantil tardia
}

\author{
Metachromatic Leukodystrophy: case report of child with \\ late infantile form
}

\section{Leucodistrofia Metacromática: presentación de um caso del ninõ con forma infantil tardía}

\author{
Murilo Henrique Berto ${ }^{1}$, João Carlos Cervelin², Rafael Frizon ${ }^{3}$, \\ Josiani Berto ${ }^{4}$
}

\begin{abstract}
1.Discente do curso de Medicina da Universidade do Planalto Catarinense (UNIPLAC), Lages-SC, Brasil. https://orcid.org/0000-0002-6352-6478

2.Discente do curso de Medicina da Universidade do Planalto Catarinense (UNIPLAC), Lages-SC, Brasil. https://orcid.org/0000-0001-7402-2737

3.Neurologista, Docente do curso de Medicina da Universidade do Planalto Catarinense (UNIPLAC), LagesSC, Brasil. https://orcid.org/0000-0002-6311-5126

4.Médica, Mestre, Docente do curso de Medicina da Universidade do Planalto Catarinense (UNIPLAC), Lages-SC, Brasil. https://orcid.org/0000-0002-3543-6862
\end{abstract}

\section{Resumo}

Introdução. Leucodistrofia Metacromática (LDM) é uma doença neurológica desmielinizante causada por um erro inato do metabolismo, resultando em deficiência da enzima arilsulfatase A. Os primeiros sinais da doença iniciam com perda dos marcos do desenvolvimento e regressão motora. Objetivo. Relatar o caso de uma criança com LDM, destacando as crises convulsivas e déficit visual no início da doença. Método. Relato de Caso de uma paciente atendida em um hospital estadual de Santa Catarina. As informações da paciente foram obtidas através de revisão do prontuário eletrônico, consultas ambulatoriais, visita domiciliar e exame físico. Resultados. A criança desenvolvia-se normalmente até o início dos sintomas. A partir do segundo ano de vida, surgiram episódios agudos de febre associados a convulsões generalizadas frequentes. Durante a internação, teve regressão do desenvolvimento neuropsicomotor e, posteriormente, déficit visual. Com o passar dos anos apresentou postura em opistótono, incapacidade de comunicação e total dependência dos pais. A criança era internada com frequência devido pneumonia e estava em cuidados paliativos. O óbito ocorreu aos 7 anos de idade. Conclusão. O quadro clínico da menina é semelhante a outros casos reportados na literatura, mas as crises convulsivas generalizadas e o déficit visual súbito foram únicos.

Unitermos. Leucodistrofia Metacromática; comprometimento; deficiência

\begin{abstract}
Introduction. Metachromatic Leukodystrophy (MLD) is a demyelinating neurological disease caused by an inborn error of metabolism, resulting in deficiency of the enzyme arylsulfatase A. The early signs of disease begin with loss of developmental milestones and motor regression. Objective. Report the case of a child with MLD, highlighting the seizures and visual deficit at the onset of the disease. Method. Case report of a patient seen at a state hospital in Santa Catarina. The patient's information was obtained through the review of the electronic medical record, outpatient consultations, home visits and physical examination. Results. The child developed normally until the onset of symptoms. From the second year of life, there were acute episodes of fever associated with frequent generalized seizures. During hospitalization, neuropsychomotor development regressed and, subsequently, visual impairment. Over the years, he presented an opisthotonic posture, inability to communicate and total dependence on his parents. The child was frequently hospitalized due to pneumonia and was in palliative care. Death occurred at 7 years of age. Conclusion. The girl's clinical condition is like other cases reported in the literature, but the generalized seizures and sudden visual deficit were unique.
\end{abstract}

Keywords. Metachromatic Leukodystrophy; impairment; deficiency 


\section{Resumen}

Introducción. La leucodistrofia metacromática (LDM) es una enfermedad neurológica desmielinizante causada por un error innato del metabolismo, que resulta en deficiencia de la enzima arilsulfatasa A. Los primeros signos de la enfermedad comienzan con la pérdida de los hitos del desarrollo y la regresión motora. Objetivo. Reporte el caso de un niño con LDM, destacando convulsiones y discapacidad visual al inicio de la enfermedad. Método. Reporte de caso de un paciente atendido en un hospital estatal de Santa Catarina. La información del paciente se obtuvo mediante la revisión de la historia clínica electrónica, consultas externas, visitas domiciliarias y exploración física. Resultados. El niño se desarrolló normalmente hasta la aparición de los síntomas. A partir del segundo año de vida, se presentaron episodios agudos de fiebre asociados a frecuentes convulsiones generalizadas. Durante la hospitalización, el desarrollo neuropsicomotor retrocedió y, posteriormente, la discapacidad visual. A lo largo de los años, presentó postura opistotónica, incapacidad para comunicarse y dependencia total de sus padres. El niño fue hospitalizado con frecuencia por neumonía y se encontraba en cuidados paliativos. La muerte ocurrió a los 7 años de edad. Conclusión. El cuadro clínico de la niña es similar a otros casos reportados en la literatura, pero las convulsiones generalizadas y el déficit visual repentino fueron únicos.

Palabras clave: Leucodistrofia metacromática; deterioro; Deficiência

Trabalho realizado Universidade do Planalto Catarinense (UNIPLAC), Lages-SC, Brasil.

Conflito de interesse: não Recebido em: 17/10/2019 Aceito em: 29/07/2020

Endereço para correspondência: Murilo H Berto. UNIPLAC, Centro de Ciência da Saúde, Departamento de Medicina. Av. Castelo Branco, 170, Bairro Universitário, CEP 88509-900. Lages-SC, Brasil. E-mail: muriloberto@gmail.com

\section{INTRODUÇÃO}

As leucodistrofias são um grupo de mais de 30 doenças genéticas heterogêneas causadas por defeitos na síntese ou metabolismo de mielinas levando a hipomielinização. A leucodistrofia metacromática (LDM), um dos tipos mais comuns de leucodistrofia, é uma doença neurológica desmielinizante causada por um erro inato do metabolismo, com uma prevalência de $1: 40.000$ a $1: 160.000$ nascidos vivos $^{1,2}$. A LDM ocorre por uma mutação autossômica recessiva do cromossomo $22 \mathrm{q}$ que resulta na deficiência da enzima arilsulfatase A (ARSA), uma enzima lisossomal que degrada diversos lipídios com grupos sulfato (sulfatídeos) ${ }^{3,4}$. 
Os sulfatídeos são os principais lipídios da mielina e são essenciais para sua estrutura e função. No entanto, quando em excesso, tem ações ações biológicas que podem inibir a diferenciação de oligodendrócitos e gerar uma resposta próinflamatória da micróglia e dos astrócitos, causando instabilidade da mielina e desmielinização central, simétrica e progressiva ${ }^{5}$. Além disso, os sulfatídeos são levados da corrente sanguínea para outros tecidos, formando depósitos nos nervos periféricos com consequente desmielinização nos neurônios periféricos ${ }^{6}$. O acúmulo de sulfatídeos também pode ocorrer em outros tecidos do corpo, como rins, bexiga e vesícula biliar ${ }^{3}$. Clinicamente, as alterações neurológicas ocorrem devido a processos de desmielinização (formação inadequada da bainha de mielina) quanto de desmielinização (perda da mielina previamente formada), com predominância significativa da última?

A classificação da doença é feita de acordo com a idade de início dos sintomas, podendo variar desde dos 18 meses de idade até a vida adulta ${ }^{8}$. A Forma Infantil Tardia corresponde à maioria dos casos, com uma prevalência de 50 a 60\%. Esta forma mostra-se a de pior prognóstico, com manifestações anteriores a 30 meses de vida ${ }^{9}$. Os sintomas iniciais são a perda dos marcos do desenvolvimento, dificuldade de marcha, disartria e disfagia. Posteriormente ocorre evolução inexorável para disfunção motora, descerebração, tetraparesia e, em alguns casos, paralisia bulbar e atrofia do nervo óptico7,10. O quadro evolui para perda total de realização de qualquer atividade, estimando 
uma sobrevida de 6 meses a 8 anos após o surgimento dos sintomas iniciais ${ }^{9}$. A Forma Juvenil corresponde a $30 \%$ dos casos e manifesta-se entre 2 e 16 anos com anormalidades de postura, emocionais e comportamentais. Pode haver quadriparesia espástica e regressão da linguagem. Por fim, a Forma adulta corresponde a $10 \%$ dos casos e é o subtipo mais raro, com início variando amplamente entre os 15 aos 60 anos. Apresenta deterioração cognitiva no início do quadro com posterior transtorno de marcha, ataxia e neuropatia periférica7. A progressão tende a ser mais lenta que nas outras formas ${ }^{9}$.

Atualmente não existe tratamento eficaz para LDM. Busca-se controlar os sintomas com analgésicos e antiinflamatórios e prevenir condições associadas. É essencial o acompanhamento multiprofissional, com médicos, fisioterapeutas, nutricionistas e terapia ocupacional.

Devido à escassez de casos descritos no Brasil, nosso objetivo foi relatar o caso de uma criança com LDM, destacando suas crises convulsivas e déficit visual no início da doença.

\section{MÉTODO}

Trata-se de um relato de caso realizado em um hospital estadual de Santa Catarina. A paciente era uma menina de 7 anos, diagnosticada com LDM de forma infantil tardia. 0 relato foi aprovado pelo Comitê de Ética em Pesquisa da 
Universidade do Planalto Catarinense em Agosto de 2019, no 15635019.1.0000.5368.

As informações da paciente foram obtidas através de revisão do prontuário eletrônico, consultas ambulatoriais e visita domiciliar no ano de 2019, nas quais avaliamos as primeiras manifestações clínicas, internações hospitalares, resultados de exames, idade do diagnóstico, patologias associadas, cirurgias realizadas, início e manutenção do tratamento. Os pais da paciente permitiram registro fotográfico dos laudos de exames realizados pela paciente para o diagnóstico da patologia. Realizou-se semiologia neurológica para avaliar o equilíbrio, pares cranianos, marcha, força, tônus, coordenação, reflexos motores e sensibilidade. Foi feita ainda semiologia osteoarticular de extremidade superior, inferior e coluna vertebral devido as deformidades ósseas e contraturas presentes. A semiologia ocular foi realizada para avaliar a acuidade visual. Comparamos ainda os dados obtidos nas entrevistas com os pais e no exame físico com os registros anteriores do prontuário eletrônico da paciente.

\section{RELATO DO CASO}

Descrevemos o caso de uma menina com LDM Infantil Tardia. Sexo feminino, branca, natural de Lages/SC. Filha de pais sem consanguinidade, veio a óbito aos 7 anos de idade. História obstétrica: pré-natal iniciado no primeiro trimestre, com realização de 10 consultas, baixo risco gestacional, sem 
exposição a teratógenos ou infecções. O parto aconteceu naturalmente, a termo, sem intercorrências em um hospital da cidade, em 21/05/2012. Nasceu com 3 kg, $47 \mathrm{~cm}$ de comprimento e Apgar 8 e 9. História pediátrica: desenvolvimento neuropsicomotor (DNPM) evoluindo com a idade. História Patológica Pregressa: Acrocianose, forame oval patente e displasia do desenvolvimento do quadril (DDQ) congênitos. A sua DDQ foi a subluxação do quadril. Meningite aos 3 meses e esofagite grau B (classificação de Los Angeles).

Aos nove meses de vida, mantinha-se sentada sozinha com a coluna ereta. Iniciou movimento de pinça com 0 indicador e polegar e falava algumas palavras aos 12 meses. Sempre apresentou distúrbio de marcha devido a DDQ.

Aos dois anos, falava algumas frases curtas com sentido, boa acuidade visual e auditiva, alimentava-se independentemente. Porém, nessa idade, surgiram episódios agudos de febre associados a convulsões generalizadas frequentes, justificando a internação em leito de Unidade de Terapia Intensiva em um hospital local. Durante a internação, apresentou regressão do desenvolvimento neuropsicomotor (DNPM). Devido a isso, após melhora parcial dos sintomas, no sétimo dia de internação, recebeu alta hospitalar, sendo encaminhada para referência em neurologia pediátrica, em um hospital do Estado de Santa Catarina. A primeira consulta no serviço especializado demorou cerca de um ano para acontecer devido à falta de 
vagas para consultas com especialistas pelo serviço público de saúde.

Aos três anos, apresentou dificuldade em falar, deglutir, choro excessivo, perda da acuidade visual e déficit cognitivo. Suspeitou-se de déficit visual devido a criança não levar a mão para pegar objetos, não estabelecer contato visual e perda do reflexo palpebral bilateralmente. Após consultas ao serviço de neurologia infantil, foram realizados exames laboratoriais e de imagem. Os laudos revelaram baixa atividade de arilsulfatase A nos leucócitos, e, através da Ressonância Magnética (RM), áreas difusas hiperintensas na substância paraventricular e centros semi-ovais, relacionando-se à gliose ou leucomalácia. Dessa forma, com as manifestações clínicas e os resultados dos exames chegou-se ao diagnóstico de LDM da forma Infantil Tardia. O próprio serviço especializado fez a referência à Unidade Básica de Saúde (UBS) do Município de Lages. A UBS encaminhou a criança para APAE (Associação de Pais e Amigos dos Excepcionais) e ao PAPS (Programa de Atenção Psicossocial) para receber atenção especializada.

Aos quatro anos, desenvolveu hipertonia apendicular e hipotonia axial, encurtamento muscular, deformidades osteomusculares, afasia, afonia, tetraplegia e postura em opistótono. Manteve episódios de crises convulsivas generalizadas. Devido a impossibilidade de alimentação via oral e perda de peso constante, necessitou de gastrostomia para regularizar a alimentação e hidratação. Fez uso 
contínuo de Ácido Valpróico e Fenobarbital para tratamento das crises convulsivas generalizadas.

Aos cinco anos, a menina já estava restrita ao leito domiciliar. Apresentou profunda regressão neuropsicomotora, não demonstrava expressão facial, incapacidade de expressar qualquer forma comunicação, totalmente dependente dos pais para alimentação e higiene. Notou-se comprometimento do sistema respiratório, com dificuldade para tossir e deglutir secreções nasofaríngeas, causando pneumonia aspirativa de repetição. Os pais foram orientados a oferecer cuidados paliativos.

Aos seis anos, devido à situação clínica, os pais optaram por não rematriculá-la na APAE e na creche. Porém, foram mantidos os tratamentos de fisioterapia respiratória domiciliar. Nesse ano conhecemos a paciente e seus pais e realizamos consultas e visitas domiciliares. Em nosso exame físico neurológico: crânio e coluna sem alterações a inspeção e palpação, membros superiores e inferiores em flexão e hipertonia generalizada, inclinação lateral da cabeça a esquerda, sinal de Babinski no pé direito, reflexo motor presente ao estímulo doloroso e reflexos profundos abolidos. Exame osteoarticular: quadriparesia espástica com deformidade estruturada dos tornozelos e punhos, incapacidade de alternar as posições de decúbito. Exame ocular: pupilas não fotorreagentes, não realiza movimentos oculares por confrontação, não segue objetos, não exibe reação de acomodação visual, reflexo palpebral ausente bilateralmente, não apresenta nistagmo. 
Aos 7 anos, no dia 31 de julho de 2019, a menina apresentou desconforto respiratório agudo, tosse e febre, sendo levada a UBS do seu bairro. Porém, os médicos constataram parada cardíaca, e iniciaram a reanimação. Acionaram o SAMU, porém sem resultados. A criança veio a óbito, sendo broncopneumonia a causa da morte.

\section{DISCUSSÃO}

A LDM pode manifestar-se clinicamente em várias idades, de forma bastante heterogênea ${ }^{9}$. Nossa paciente apresentou DNPM normal até atingir o segundo ano de vida. Em seguida, surgiram os primeiros sintomas de disfunção neurológica e somente aos 3 anos foi feito o diagnóstico definitivo. Pesquisas descrevem variação de início dos sintomas entre 11 meses de vida até 27 anos e demora de até 4 anos para diagnóstico ${ }^{1,13}$. Esse tempo é explicado pelas manifestações clínicas inespecíficas e numerosos diagnósticos diferenciais.

Em relação ao início da manifestação clínica, nossa paciente apresentou episódios agudos de febre, convulsões generalizadas e déficit visual súbito, características incomuns no início da doença.

A crise convulsiva manifesta-se por contrações musculares anormais e excessivas, devido a descargas neuronais desordenadas e paroxísticas de um grupo de neurônios corticais. As principais causas em crianças incluem crises febris, epilepsia, infecções do sistema nervoso central 
(SNC), distúrbios eletrolíticos, erros inatos do metabolismo, traumatismo cranioencefálico, entre outros ${ }^{14}$. A atrofia óptica geralmente está presente na resposta pupilar diminuída a estímulos luminosos, caracterizando falência visual ${ }^{15}$.

De acordo com estudos, os primeiros sinais da doença iniciam-se com regressão motora e perda dos marcos do desenvolvimento, sendo estes os maiores motivos de busca por consultas em serviços especializados ${ }^{1,6,10,16-18}$. Em um relato de caso catarinense, meninas gemelares iniciaram quadro de febre com posterior déficit motor, mas sem a ocorrência de convulsões ${ }^{19}$. Outro estudo descreveu uma paciente com mudanças comportamentais aos 16 anos, isolamento acentuado, desempenho e comportamento ruim na escola, padrão de sono alterado, alucinações e comportamento desorganizado. Isso levou a um diagnóstico inicial de esquizofrenia paranoide ${ }^{3}$. Além disso, um relato de 4 pacientes revela quadros de agressividade, desinibição, delírios e síndrome de Asperger antes do diagnóstico de $\mathrm{LDM}^{13}$. Quando a função motora está intacta, os sintomas podem ser similares a esquizofrenia, depressão, dificuldade no aprendizado e transtorno do espectro autista ${ }^{13}$.

A idade de início dos sintomas tende a ser semelhante nos casos familiares. A paciente descrita nesse estudo possui um irmão de 16 anos que não apresentou sinais da doença. Como a LDM é herdada de forma autossômica dominante, cada irmão de um indivíduo com LDM terá $25 \%$ de chance de ser afetado, $50 \%$ de chance de ser portador assintomático e $25 \%$ de não ser afetado e não ser portador ${ }^{8}$. Avaliamos 
estudo de 3 crianças brasileiras de um casamento consanguíneo de primos de primeiro grau ${ }^{16}$. Nesse caso, os pais são portadores e todos os filhos desenvolveram a doença.

$\mathrm{Na}$ investigação complementar da doença, exames de imagem, como a RM em ponderações T2 e FLAIR, são úteis para o diagnóstico, facilitando inclusive o diagnóstico diferencial7,10. A RM demonstra desmielinização de forma simétrica e confluente. Acredita-se que as primeiras alterações ocorram no corpo caloso, seguido das áreas periventriculares e centro semioval, corroborando com 0 laudo do exame da nossa paciente. Além disso, o comprometimento na forma infantil tardia ocorre inicialmente nos lobos occipitais, seguindo uma direção anterior, ao contrário das outras duas formas com início frontal e progressão antero-posterior ${ }^{7}$. Em nossa paciente, assim como em outras pesquisas, analisou-se a RM antes da

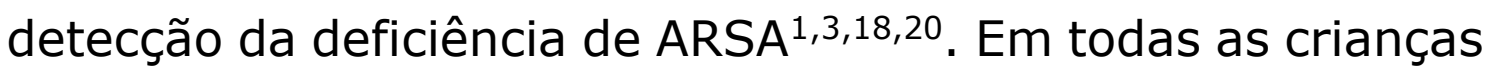
estudadas, a atividade da ARSA em leucócitos foi testada para diagnóstico, exceto em uma pesquisa, onde a análise urinária revelou aumento dos sulfatídeos, diagnosticando $\operatorname{LDM}^{20}$.

O aconselhamento genético familiar é fundamental quando a LDM é diagnosticada, podendo prevenir novos casos. Não foram realizados testes genéticos para avaliar a suscetibilidade familiar devido ao alto custo e a falta de recursos dos pais, porém em pesquisas estrangeiras 
realizou-se o exame, revelando heterozigose dos pais ${ }^{1,3,13,18}$. Não encontramos relatos brasileiros com testes genéticos.

A necessidade de cirurgia nas crianças com LDM é comum, principalmente por disfunção da musculatura lisa e da estriada esquelética. Nossa paciente foi submetida a tratamento da DDQ e gastrostomia. Estudo goiano relata duas crianças com LDM que também realizaram cirurgias como a de refluxo gastroesofágico, gastrostomia, traqueostomia e de tenotomia de adutores dos quadris para melhorar a qualidade de vida ${ }^{16}$. Chama atenção o relato de Papilomatose da vesícula biliar em portadora de LDM, necessitando de cirurgia de emergência devido hemorragia digestiva alta e choque hipovolêmico ${ }^{21}$.

O comprometimento do sistema respiratório na LDM é comum devido dificuldade em tossir, deglutir e engasgos frequentes. Nossa paciente foi internada por pneumonias de repetição e broncopneumonia por aspiração. As duas crianças do estudo goiano foram internadas em UTI devido a insuficiência respiratória e seus prognósticos de vida são desfavoráveis mesmo com o acompanhamento multidisciplinar (ortopedista, fisiatra, pneumologista, neurologista) desde o início do quadro ${ }^{16}$. Geralmente a causa do óbito é a broncopneumonia ${ }^{6}$.

Por outro lado, é notável que a sobrevida dos pacientes com LDM vem aumentando consideravelmente. A sobrevida em 5 anos, após o diagnóstico, dos casos relatados após o ano de 1990 é significativamente maior do que os casos de 1970 . Os dados demonstram sobrevida de $52 \%$ 
para a forma infantil tardia, 100\% para juvenil e 95\% para a adulta, entre 1990 a 2006. Em contraste, 14\%, 46\% e 67\% respectivamente, no período de 1970 . Percebe-se que a forma infantil tardia é a mais letal, no entanto houve melhoras significativas de todas as formas clínicas da doença nos últimos anos ${ }^{20}$.

Atualmente, a LDM é uma doença sem cura. A forma infantil tardia, da nossa paciente, é a de prognóstico mais desfavorável. O tratamento é paliativo e as limitadas opções terapêuticas disponíveis visam retardar o curso clínico da doença. Deve-se oferecer medidas de suporte para melhorar a qualidade de vida, envolvendo médicos, fisioterapeutas, fonoaudiólogos, nutricionistas, psicólogos, a fim de atenuar as complicações da doença, maximizar as funções intelectuais e neuromusculares e trazer conforto para a paciente.

\section{CONCLUSÃO}

Descrevemos um relato de caso da forma infantil tardia da LDM. Segundo a literatura médica, é a apresentação clínica mais comum e a de pior prognóstico da doença. No entanto, a sobrevida desses pacientes vem aumentando pela melhor abordagem prognóstica.

O quadro clínico da nossa paciente é compatível às descrições científicas. Porém, devido às crises convulsivas generalizadas e o déficit visual súbito antecederem a 
regressão clínica, nota-se singularidade em comparação aos outros relatos.

\section{REFERÊNCIAS}

1.Álvarez-Pabón Y, Lozano-Jiménez JF, Di Lizio-Miele KG, ContrerasGarcía GA. Leucodistrofia metacromática infantil tardía: Presentación de un caso. Arch Argent Pediatr 2019;117:52-5. http://dx.doi.org/10.5546/aap.2019.e52

2.Ortega-cruz L, Olguín-Escobar E, Martínez-Olguín M. Tratamiento fisioterapéutico en paciente pediátrico con leucodistrofia metacromática. Reporte de caso. Rev Fisioterap Tec Med 2017;1:3540.

https://www.ecorfan.org/taiwan/research_journals/Fisioterapia/vol1n um2/Revista de Fisioterapia y Tecnolog\%C3\%ADa M\%C3\%A9dica V1 N2 6.pdf

3.Espejo LM, de la Espriella R, Hernández JF. Metachromatic leukodystrophy. Case presentation. Rev Colomb Psiquiatr 2017;1:449. https://doi.org/10.1016/j.rcp.2016.05.001

4. Kumar V, Abbas AK, Aster JC. Robbins \& Cotran Patologia - Bases Patológicas das Doenças. 9a ed. Rio de Janeiro: Elsevier, 2016, 1440p. 5. Behrman RE, Jenson HB, Kliegman R. Nelson. Tratado de Pediatria. 20a ed. Rio de Janeiro: Elsevier, 2017, 3896p.

6. Nascimento OJM, Freitas MRG, Alencar AA, Couto BHN. Leucodistrofia metacromática: registro de um caso. Arq Neuropsiquiatr 1980;38:28892. http://dx.doi.org/10.1590/S0004-282X1980000300009

7.Artigalás 0 . Leucodistrofa metacromática: caracterização epidemiológica, bioquímica e clínica de pacientes brasileiros (Dissertação). Porto Alegre: Universidade Federal do Rio Grande do Sul, 2009, 141p.

https://www.lume.ufrgs.br/bitstream/handle/10183/29560/00070345 0 .pdf? sequence $=1$ \&isAllowed $=y$

8. Rosenberg JB, Kaminsky SM, Aubourg P, Crystal RG, Sondhi D. Gene therapy for metachromatic leukodystrophy. J Neurosci 2016;94:116979. https://doi.org/10.1002/jnr.23792

9.Gomez-Ospina, N. Arylsulfatase A Deficiency. Genereviews 2017;1:1-24.

10. Hidalgo R, Villarroel M. Leucodistrofia Metacromatica Infantil Tardia. Rev Chil Pediatr 1985;56:176-80.

http://dx.doi.org/10.4067/S0370-41061985000300009

11.Gieselmann V, Krägeloh-Mann I. Metachromatic Leukodystrophy An Update. Neuropediatrics 2010;41:1-6. https://doi.org/10.1055/s0030-1253412

12.Bonkowsky JL, Wilkes J, Bardsley T, Urbik, VM, Stoddard G. Association of Diagnosis of Leukodystrophy With Race and Ethnicity 
Among Pediatric and Adolescent Patients. JAMA Netw Open 2018;1:17. http://doi.org/10.1001/jamanetworkopen.2018.5031

13.Van Rappard DF, de Vries ALC, Oostrom KJ, Boelens JJ, Hollak CEM, van der Knaap MS, et al. Slowly Progressive Psychiatric Symptoms: Think Metachromatic Leukodystrophy. Jaacap 2018;57:74-6. https://doi.org/10.1016/j.jaac.2017.11.017

14.Burns DAR, Júnior DC, Silva LR, Borges WG. Tratado de pediatria: Sociedade Brasileira de Pediatria. 4a ed. Barueri: Manole, 2017, 2472 p.

15.Vilanova LCP, Santos LMG. Doença de Krabbe (leucodistrofia célula globóide): relato de um caso. J Pediatr 1998;74:153-6. http://www.jped.com.br/conteudo/98-74-02-153/port.pdf

16.Ribeiro EQ, Ribeiro MFM. Leucodistrofia Metacromática: Relato de Caso de Dois Irmãos Consanguíneos. Rev Neurocienc 2013;4:5806. https://doi.org/10.34024/rnc.2013.v21.8152

17. Werneck LM, Pereira JLP, Bruck I. Leucodistrofa metacromática: relato de dois casos com histoquímica de nervos e músculos. Arq Neuropsiquiatr 1980;38:237-48. http://dx.doi.org/10.1590/S0004282X1980000300003

18. Kolnikova M, Jungova P, Skopkova M, Foltan T, Gasperikova D, Mattosova $S$, et al. Late Infantile Metachromatic Leukodystrophy Due to Novel Pathogenic Variants in the PSAP Gene. J Mol Neurosci 2019;11:559-63. https://doi.org/10.1007/s12031-019-1259-7

19.Wittig EO, Marçallo FA, Pilotto RF, Mello LR. Leucodistrofa metacromática infantil em gêmeos. Arq Neuropsiquiatr 1985;43:3314. http://dx.doi.org/10.1590/S0004-282X1985000300016

20. Mahmood A, Berry J, Wenger DA, Escolar M, Sobeih M, Raymond G, et al. Metachromatic Leukodystrophy: a Case of Triplets with the Late Infantile Variant and a Systematic Review of the Literature. J Child Neurol 2010;25:572-80.

https://dx.doi.org/10.1177\%2F0883073809341669

21.Leite RFG, Lima GJS, Abras GM, Pires LJS, Castro EG, Bomfim GM. Papilomatose da vesícula biliar associada à leucodistrofia metacromática, uma causa rara de hemobilia não traumática. Ged Gastroenterol Endosc 2013;32:120-2.

http://files.bvs.br/upload/S/0101-7772/2013/v32n4/a5008.pdf 